\title{
Augmenter le taux de vaccination contre le SRAS-CoV-2 parmi la population noire au Canada
}

\author{
Azza Eissa MD PhD, Aisha Lofters MD PhD, Nancy Akor BSclnf, Cheryl Prescod MSc, Onye Nnorom MD MSP
}

Citation : CMAJ 2021 August 9;193:E 1220-1. doi : 10.1503/cmaj.210949-f

Voir la version anglaise de l'article ici : www.cmaj.ca/lookup/doi/10.1503/cmaj.210949

E n juin 2020, Santé publique Ontario a amorcé la collecte de données sur la race des patients atteints de la COVID-19, après des mois de plaidoyer de la part de la communauté. Le caractère disproportionné du taux d'infection des personnes noires au SRAS-CoV-2 dans la province est rapidement devenu apparent. Selon les premières données dévoilées par le Bureau de santé publique de Toronto, les populations de descendances africaine et caribéenne comptent pour $21 \%$ des cas de COVID-19 dans la ville alors qu'elles ne représentent que $9 \%$ de sa population ${ }^{1}$. Pour les mois de janvier à mai 2020, les taux d'infection au SRAS-CoV-2, d'hospitalisation, d'admission aux soins intensifs et de décès étaient respectivement 3, 4, 4 et 2 fois supérieurs à la moyenne globale dans les quartiers les plus multiethniques de l'Ontario ${ }^{2}$. Dans les populations noires de Toronto, le taux d'infection à la COVID-19 a progressé de 741 cas à 1311 cas par 100000 habitants entre les mois de mars et avril $2021^{1}$. Bien qu'ils soient parmi les plus durement touchés par la pandémie, seulement $56,6 \%$ des Noirs canadiens ont indiqué vouloir recevoir le vaccin contre le SRASCoV-2, comparativement à $76,9 \%$ pour la population générale ${ }^{3}$, selon un rapport de Statistique Canada publié en mars 2021. L'Organisation mondiale de la Santé définit la réticence à l'égard de la vaccination comme un délai dans l'acceptation ou le refus des vaccins malgré la disponibilité de services de vaccination ${ }^{4}$. Ainsi, avant de cataloguer des communautés et des patients comme étant réticents à la vaccination ou peu éduqués en ce qui concerne la santé, nous devons nous assurer qu'un accès adéquat aux services de vaccination est offert et de remédier au racisme systémique qui explique un grand nombre d'inégalités sociales auxquelles font face les communautés noires au Canada. Au cours d'une pandémie qui a éprouvé ces communautés de façon disproportionnée, la vaccination contre le SRAS-CoV-2 donne l'occasion de

\section{Points clés}

- Les personnes noires du Canada courent un risque élevé de contracter l'infection au SRAS-CoV-2, d'être hospitalisées, d'être admises aux soins intensifs et de décéder de la COVID-19; malgré cela, elles sont plus réticentes à se faire vacciner que les autres Canadiens.

- La réticence à l'égard de la vaccination dans les communautés noires n'est pas seulement une question de mésinformation ou de manque d'éducation en matière de santé : elle est associée à une méfiance à l'égard des institutions médicales et au racisme structurel.

- Des approches afrocentristes de promotion de la santé et de conseil qui sont centrées sur le respect des valeurs et des points de vue des patients se sont avérées efficaces pour améliorer le taux de vaccination contre la grippe et le SRASCoV-2 parmi les populations noires du Canada.

- Le cadre de communication LEAPS of care (Listen and Learn, Empower and Engage, Ask and Acknowledge, Paraphrase and Provide, Support and Spark) peut aider les cliniciens à faire tomber les barrières et ainsi à augmenter la vaccination chez les patients noirs réticents.

- Les partenariats menés par des Noirs qui allient le secteur des soins de santé et des intervenants ayant déjà établi des relations de confiance dans la communauté peuvent augmenter la confiance à l'égard de la vaccination contre le SRAS-CoV-2 dans les communautés noires en confrontant le racisme anti-noir et en élargissant la sensibilisation.

rétablir la confiance et l'imputabilité et de corriger les inégalités de longue date vécues par les populations noires lorsqu'il est question de santé. Nous discutons ici de ce que les cliniciens peuvent faire pour tenter d'augmenter le taux de vaccination contre le SRAS-CoV-2 chez les Noirs canadiens.

Bien qu'aucune donnée probante n'ait démontré l'existence d'une réticence à l'égard de la vaccination systématique des 
enfants parmi les populations noires au Canada, une étude de 2012 a montré que les Noirs de l'Ontario constituaient le groupe racisé le moins susceptible de se faire vacciner contre la grippe ${ }^{5}$. Le centre de santé communautaire TAIBU, qui dessert des populations principalement noires, a élaboré un modèle pour s'attaquer à cet enjeu. Grâce à une initiative d'amélioration continue centrée sur le respect du point de vue des Noirs pour accroître la confiance à l'égard de la vaccination, le centre a augmenté le taux de vaccination contre la grippe parmi les populations qu'il dessert, le faisant passer de $8 \%$ en 2013 à $53 \%$ en $2018^{6}$. Les prestataires de soins du centre TAIBU ont employé une approche " afrocentriste » de promotion de la santé, ancrée dans des valeurs de coopération et de contributions collectives $^{7,8}$. Le terme " afrocentrisme » englobe les populations de descendances africaine et caribéenne, ce qui signifie que les prestataires de soins plaçaient cette communauté au centre de leur travail. Ils écoutaient les patients et parlaient avec eux de façon respectueuse, reconnaissaient leurs préoccupations fondées sur des valeurs et des croyances et dégageaient les freins à la vaccination. Le centre tentait ensuite de lever ces freins en mettant de l'avant des ressources et des affiches adaptées et propres à la culture sur le vaccin contre la grippe, en allongeant les heures d'ouverture de la clinique, en utilisant un système de rappel de rendez-vous pour les patients et en informant ces derniers que les prestataires de soins étaient eux-mêmes vaccinés. Nous suggérons aux cliniciens d'intégrer une approche afrocentriste comparable pour augmenter la vaccination contre le SRAS-CoV-2 parmi les Noirs canadiens.

Une approche afrocentriste, qui reconnaît aussi que les expériences de soins des populations noires sont influencées par le racisme anti-noir, historique comme contemporain ${ }^{9,10}$, peut être combinée à des cadres de dialogue avec les patients qui éprouvent des réticences à l'égard de la vaccination. Par exemple, les prestataires pourraient employer le modèle LEAPS of care : écouter et apprendre du vécu des patients; les responsabiliser et les mobiliser en respectant leur autodétermination et leurs points de vue; les interroger et reconnaître leurs peurs et leurs préoccupations, notamment s'informer de leurs interactions antérieures avec les soins de santé, durant lesquelles ils peuvent avoir subi du racisme; reformuler et fournir des renseignements sur la vaccination et des documents d'information en plus d'administrer le vaccin si le patient y consent; soutenir et susciter la participation des patients et les partenariats communautaires futurs, au besoin.

Lorsqu'ils fournissent des renseignements, les cliniciens peuvent rehausser la confiance des Noirs canadiens en citant des données sur le nombre de patients noirs vaccinés et souligner la contribution des scientifiques noirs, comme le $D^{r}$ Anthony Fauci l'a fait en mentionnant que le vaccin de Moderna "a été élaboré par une Afro-américaine (la D re Kizzmekia Corbett) ${ }^{11}$. L'appui du produit par les médecins et les infirmiers est l'un des facteurs les plus importants dans les décisions vaccinales de tous les patients ${ }^{11}$. Il faut transmettre des renseignements clairs, cohérents et accessibles sur l'efficacité du vaccin, ses contre-indications, ses effets indésirables et le délai de protection (avec les multiples doses) pour accroître la confiance à l'égard des vaccins. Insister sur l'importance d'une couverture vaccinale à l'échelle de la population pour protéger la communauté peut aussi contribuer à accroître l'adhésion à la vaccination.

Il est important que les cliniciens non seulement transmettent des renseignements sur le vaccin, mais aussi qu'ils soutiennent les patients en les orientant dans le système complexe des soins de santé. Les contradictions au sujet des variants du SRAS-CoV-2, de la sécurité des vaccins, des événements indésirables, des groupes prioritaires et des sites de vaccination (p. ex., entre un site Web de vaccination et les messages du gouvernement) ont nui à la confiance dans les vaccins. Cette confiance ne s'améliorera pas si les communautés noires se font dire qu'elles présentent un risque élevé et qu'elles doivent poursuivre les mesures de distanciation physique alors qu'elles sont en même temps exclues des groupes prioritaires ou qu'on n'augmente pas l'accessibilité de la vaccination pour elles. Les prestataires devraient offrir aux patients noirs à haut risque des renseignements exacts et à jour sur la manière de recevoir les vaccins, étant donné l'évolution constante des critères de préinscription aux différents sites. Les partenariats de santé menés par des Noirs jouent un rôle central dans la résolution de ces problèmes. Par exemple, le centre de santé communautaire Black Creek (situé au nord-ouest de Toronto, un secteur très touché par la pandémie) a fait appel à des ambassadeurs communautaires formés et a coordonné une série de séances de vaccination à faibles contraintes les soirs et les fins de semaine pour accommoder les travailleurs essentiels, ce qui a entraîné une hausse fructueuse du taux de vaccination, qui est passé de 5,5\% en avril 2021 à $56,3 \%$ en mai $2021^{1}$

Les centres de santé communautaires (p. ex., TAIBU et Black Creek), les organismes dirigés par des Noirs (p. ex., la Black Health Alliance, le Black Scientists' Task Force on Vaccine Equity, l'Association des médecins noirs de l'Ontario, l'Association de santé des Afro-Canadiens) et de nombreux leaders noirs du milieu de la santé de la NouvelleÉcosse et d'autres provinces ont combattu la méfiance à l'égard des vaccins au sein des communautés noires en organisant des assemblées publiques, en réclamant une collecte de données sur la race et en créant des ressources à relayer qui contrent la mésinformation. Les cliniciens devraient être au courant et connaître ces ressources. Il reste du travail à faire dans la collecte minutieuse de données afin d'améliorer les solutions à la crise de la COVID19 au sein des communautés noires. Nous ne pouvons nous contenter de présenter des données épidémiologiques alarmantes sans passer à l'action ${ }^{12,13}$. Nous avons besoin de données qualitatives sur le racisme vécu par les populations noires pendant la pandémie pour soutenir les initiatives visant à faire tomber les barrières et à améliorer les expériences, l'accès et les retombées en matière de soins de santé pour les personnes noires du Canada. 


\section{Références}

1. COVID-19: Status of cases in Toronto. Ethno-racial group, income, \& infection. Toronto: City of Toronto; 2021. Accessible ici : https://www. toronto.ca/home/covid-19/covid-19-latest-city-of-toronto-news/covid-19 -status-of-cases-in-toronto/ (consulté le 23 juill. 2021).

2. COVID-19 in Ontario - a focus on diversity. Toronto: Public Health Ontario; 2021. Accessible ici : https://www.publichealthontario.ca/-/media/documents/ ncov/epi/2020/06/covid-19-epi-diversity.pdf?la=en. (consulté le 26 mai 2021).

3. COVID-19 vaccine willingness among Canadian population groups. Ottawa: Statistics Canada; 2021. Accessible ici : https://www150 .statcan.gc.ca/n1/pub/45-28-0001/2021001/article/00011eng.htm (consulté le 26 mai 2021).

4. Social determinants and inequities in health for Black Canadians: a snapshot. Ottawa: Government of Canada; modified 2020 Sept. 8. Accessible ici : https://www.canada.ca/en/public-health/services/health-promotion/ population-health/what-determines-health/social-determinants-inequities -black-canadians-snapshot.html (consulté le 26 mai 2021).

5. Quach S, Hamid J, Pereira J, et al. Influenza vaccination coverage across ethnic groups in Canada. CMAJ 2012;184:1673-81.
6. Jain A, Akor N, Nnorom O, et al. Use of a quality improvement strategy to increase flu immunization rates among racialized groups [poster]. Family Medicine forum; 2015 Nov. 11-14; Toronto.

7. Davis SK, Williams A, Akinyela M. An Afrocentric approach to building cultural relevance in social work research. J Black Stud 2010;41:338-50.

8. Hatcher SS, King DM, Barnett T, et al. Mental health for youth: applying an African-centered approach. J Hum Behav Soc Environ 2016;27:1-12.

9. Quinn SC, Andrasik M. Addressing vaccine hesitancy in BIPOC communities toward trustworthiness, partnership, and reciprocity. N Engl J Med 2021;385:97.

10. Bajaj SS, Stanford FC. Beyond Tuskegee - vaccine distrust and everyday racism. N Engl J Med 2021;384:e12.

11. Schaffer DeRoo S, Pudalov NJ, Fu LY. Planning for a COVID-19 vaccination program. JAMA 2020;323:2458-9.

12. Black HealthEquity Working Group. Engagement, governance, access, and protection (EGAP): a data governance framework for health data collected from Black communities in Ontario. Blackhealthequity.ca; 2021. Accessible ici : https://blackhealthequity.ca/wp-content/uploads/2021/03/Report_ EGAP_framework.pdf (consulté le 26 mai 2021).

13. Burgess RA, Osborne RH, Yongabi KA, et al. The COVID-19 vaccines rush: participatory community engagement matters more than ever. Lancet 2021;397:8-10.
Intérêts concurrents : Azza Eissa, Aisha Lofters et Onye Nnorom sont membres de l'Association des médecins noirs de l'Ontario. Nancy Akor est infirmière autorisée au centre de santé communautaire TAIBU. Cheryl Prescod est la directrice générale du centre de santé communautaire Black Creek. Aucun autre intérêt concurrent n'a été déclaré.

Cet article a été révisé par des pairs.

Affiliations : Département de médecine familiale et communautaire (Eissa, Lofters, Nnorom) et École de santé publique Dalla Lana (Eissa, Lofters, Nnorom), Université de Toronto; Centre Peter Gilgan pour les cancers féminins (Lofters), Hôpital Women's College; centre de santé communautaire TAIBU (Akor); centre de santé communautaire Black Creek (Prescod), Toronto, Ont.
Collaborateurs : Azza Eissa, Aisha Lofters et Onye Nnorom ont contribué à l'élaboration et à la conception du travail. Nancy Akor et Cheryl Prescod ont décrit comment les partenariats communautaires dirigés par des Noirs aux centres de santé communautaire TAIBU et Black Creek, respectivement, emploient des approches de promotion de la santé afrocentristes et utilisent les relations de confiance existantes afin d'augmenter les taux de vaccination dans les communautés desservies. Azza Eissa a rédigé le manuscrit. Toutes les auteures ont révisé de façon critique le contenu intellectuel important du manuscrit; elles ont donné leur approbation finale pour la version destinée à être publiée et assument l'entière responsabilité de tous les aspects du travail.
Propriété intellectuelle du contenu : Il s'agit d'un article en libre accès distribué conformément aux modalités de la licence Creative Commons Attribution (CC BY-NC-ND 4.0), qui permet l'utilisation, la diffusion et la reproduction dans tout médium à la condition que la publication originale soit adéquatement citée, que l'utilisation se fasse à des fins non commerciales (c.-à-d., recherche ou éducation) et qu'aucune modification ni adaptation n'y soit apportée. Voir : https://creativecommons.org/ licenses/by-nc-nd/4.0/deed.fr.

Remerciements : Les auteures remercient Sané Dube, du Centre Gattuso pour la médecine sociale du Réseau universitaire de santé, pour son aide à la révision d'une version antérieure du manuscrit.

Correspondance : Azza Eissa, azza.eissa@mail.utoronto.ca 